\title{
A study on burning behavior and convective flows in Methanol pool fires bound by ice
}

Farahani, Hamed Farmahini; Jomaas, Grunde; Rangwala, Ali S.

Published in:

2017 International Oil Spill Conference Proceedings

Link to article, DOI:

10.7901/2169-3358-2017.1.1983

Publication date:

2017

Document Version

Peer reviewed version

Link back to DTU Orbit

Citation (APA):

Farahani, H. F., Jomaas, G., \& Rangwala, A. S. (2017). A study on burning behavior and convective flows in Methanol pool fires bound by ice. In 2017 International Oil Spill Conference Proceedings (Vol. 2017, pp. 19831998). International Oil Spill Conference Proceedings Vol. 2017 No. 1 https://doi.org/10.7901/2169-33582017.1.1983

\section{General rights}

Copyright and moral rights for the publications made accessible in the public portal are retained by the authors and/or other copyright owners and it is a condition of accessing publications that users recognise and abide by the legal requirements associated with these rights.

- Users may download and print one copy of any publication from the public portal for the purpose of private study or research.

- You may not further distribute the material or use it for any profit-making activity or commercial gain

- You may freely distribute the URL identifying the publication in the public portal 
Abstract ID: 2017-170

2017 INTERNATIONAL OIL SPILL CONFERENCE

\title{
A study on burning behavior and convective flows in Methanol pool fires bound by ice
}

\author{
Hamed Farmahini Farahani $^{\mathrm{a}^{*}}$, Grunde Jomaas ${ }^{\mathrm{b}}$, Ali S. Rangwala ${ }^{\mathrm{a}}$ \\ ${ }^{\text {a }}$ Department of Fire Protection Engineering, Worcester Polytechnic Institute, Worcester, MA 01609, USA \\ b Technical University of Denmark (DTU), Department of Civil Engineering, 2800 Kgs. Lyngby, Denmark*
}

Abstract (ID: 2017-170)

An experimental study on methanol pool fires bound by ice was carried to research the burning behavior and flow field (within the liquid-phase) of methanol. The experiments were conducted in two parts: 1 - in a cylindrical ice cavity/pan (10.2 cm diameter and $6 \mathrm{~cm}$ depth) at three different conditions to analyze burning parameters of methanol, 2- in a square glass tray with outside dimensions of $10 \times 10 \mathrm{~cm}$ and a depth of $5 \mathrm{~cm}$ to obtain flow field of methanol pool with a two-dimensional PIV (Particle Image Velocimetry) system. The results of the experiments of the first part show the cold boundaries of the ice cavity/pan act as a heat sink causing considerable heat losses. Thus, burning rates and burning efficiencies are found to be lower with cold boundaries. However, the burning rate values in ice cavity are found to be the highest because of the melting of the ice and expansion of the cavity. The analysis of the results obtained by the PIV system showed the velocity magnitudes and flow patterns in the liquid-phase of icy methanol fire significantly change over the course of burning. In the instants after ignition a horizontal flow induced by Marangoni near the surface was observed. Later on, mixing of melt-water with methanol and sinking of this mixture caused a cycle in the tray that resulted in a vortex appearing in the middle of the pool. Magnitudes of velocity were also observed to increase after ignition. The increase in the velocity magnitudes is expected to significantly impact the melting and size of the lateral cavity.

Keywords: oil spill, ice, in situ burning, melting, convection.

*: current mailing address:

BRE Centre for Fire Safety Engineering, University of Edinburgh, Edinburgh, EH9 3FG, United Kingdom 


\section{INTERNATIONAL OIL SPILL CONFERENCE}

\section{INTRODUCTION}

An Arctic oil spill can interact with ice in many different ways and as a result many different methods of spill response may be necessary. In situ burning (ISB) has been found to be an effective method for oil spill cleanup in icy conditions (Buist and Dickins 1987, Ross 2003, DeCola et al. 2006, Brandvik et al. 2006, Buist et al. 2013). Interactions of the burning oil and ice during in situ burning operation after a subsurface blowout or a spill directly on ice may lead to lower clean-up/burning efficiency because of multiple reasons. Mainly, the low temperature of the ice will have an adverse effect on the burning which could impede the burning mechanisms (Vali et al. 2013). Secondly, the change in geometry of the ice and oil, because of ice melting, brings about substantial changes to the initial geometry that will affect the burning dynamics (Farahani et al. 2014, Van Gelderen et al. 2015). One of these geometry transformations that have been observed in previous studies is a phenomenon referred as lateral cavity formation. During laboratory experiments of burning of liquid fuels adjacent to ice walls, the burning fuel was observed to melt the ice and create a lateral cavity (Bellino et al. 2013, Farahani et al. 2014, Farahani et al. 2015). In addition to the adverse influences induced by the low temperatures of the ice, the formation of the lateral cavity may be a potential setback for the success of in situ burning too. For example, the ice deformation will allow a portion of the fuel to drift underneath the lateral cavity. This also could be a factor in reducing the burning efficiencies by reducing the access of air to the trapped fuel. A decrease in the burning efficiency leads to larger amounts of residue. In addition, the confined residue in the cavity would be harder to collect depending on the penetration length of the fuel into the ice and, as a result, increase the cost of the post burn clean-up during in situ burning operations. In particular, if the residue stays untreated, it could be encapsulated because of freezing of the water in colder seasons and potentially remain in the ecosystem for years.

The heat transfer mechanisms of bounded pool fires have been under investigation for decades and most of the elements of heat input/output to the liquid fuels are evaluated (Hamins 1994, Nakakuki 1994, Hamins 1999). Especially, the heat transfer through the walls of the pool for different materials (steel, glass, copper, etc.) and different diameters is studied previously (Nakakuki 1994). These studies have been focused on where the energy enters the liquid, but have 
Abstract ID: 2017-170

\section{INTERNATIONAL OIL SPILL CONFERENCE}

not addressed the subsequent redistribution of that energy within the liquid except for a few studies (Yumoto et al. 1997, Vali et al. 2014). A recent study on a methanol pool fire (Vali et al. 2014) presented flow structures within the liquid and near the rims of the pool indicating the magnitude of convection on the bulk and surface of the fuel. Further, the effects of convective flow on the walls of the pool were dismissed until a new practical problem emerged; burning of fuels spilled in ice cavities. For this reason, the influence of ice melting on liquid fuel burning behavior will be analyzed which is the topic central to the current research.

The objective of this study is to analyze the burning behavior and visualize the flow in a methanol layer using Particle Image Velocimetry (PIV) technique. Understanding the effect of ice on burning behavior and the convective flows in an icy pool fire could explain the lower burning efficiencies and lateral cavity formation. Although, melt pools from oil spill in ice appear in large size and probably involve a multi-component fuel, i.e. crude oil, the knowledge obtained by laboratory-scale experiments on pure liquid fuels are of practical and theoretical value too. Similarly, the results of this study can be used to help predict the outcomes associated with burning dynamics and flow characteristics of large-scale oil burns.

\section{EXPERIMENTAL SETUP}

The experiments were conducted in two parts to investigate the burning behavior of methanol in different conditions and the convective flows within the liquid phase of methanol pool fire bound by an ice wall. The liquid fuel used herein was methanol, with density of $792 \mathrm{~kg} / \mathrm{m}^{3}$ and a boiling point of $64.7^{\circ} \mathrm{C}$ (Yaws 1995). A schematic of the experimental setup is shown in Fig. 1. Figure 1(a) shows the sketch of the setup for the first part of the study that was used to analyze the burning behavior of a cylindrical pool of methanol. Experiments for this part of the study were conducted with burning of methanol in a cylindrical ice cavity of $10.2 \mathrm{~cm}$ diameter and $6 \mathrm{~cm}$ depth. Two sets of complementary burning experiments of methanol-water mixture were also conducted in a pan (10.2 cm diameter and $6 \mathrm{~cm}$ depth) surrounded by "room temperature water" and "ice bath". The setup was placed on a load cell (0.01 g precision) to record mass loss and a propane torch igniter was used to ignite the mixture after it was poured into the pan. Three different amounts of methanol (64.5, 97, and 129.5 g) were used for every condition to make an 


\section{INTERNATIONAL OIL SPILL CONFERENCE}

experimental matrix with total of 9 experiments. For the complementary experiments in the pan, methanol was added to a layer of water to mimic the water mixing that occurs in the ice cavity tests. Table 1 provides the details of the tests on burning behavior. The experiments were repeated three times to ensure the reproducibility of the results. The burning duration, mass loss of the methanol, and geometry change of the ice were experimentally determined.

Table 1. Experimental matrix for the burning behavior tests.

\begin{tabular}{|c|c|c|c|c|}
\hline & $\begin{array}{l}\text { Initial mass of } \\
\text { methanol (g) } \\
\text { For tests in ice } \\
\text { cavity }\end{array}$ & $\begin{array}{c}\text { Initial methanol } \\
\text { volume fraction in } \\
\text { water }\end{array}$ & $\begin{array}{c}\text { Initial } \\
\text { methanol layer } \\
\text { thickness (cm) }\end{array}$ & $\begin{array}{l}\text { Initial } \\
\text { ullage } \\
\text { (cm) }\end{array}$ \\
\hline \multirow{3}{*}{ 1- In ice cavity } & 64.5 & $100 \%-0 \%$ & 1.0 & 5.0 \\
\hline & 97 & $100 \%-0 \%$ & 1.5 & 4.5 \\
\hline & 129.5 & $100 \%-0 \%$ & 2.0 & 4.0 \\
\hline \multirow{3}{*}{ 2- Pan in water } & 64.5 & $17 \%-83 \%$ & 1.0 & 0 \\
\hline & 97 & $25 \%-75 \%$ & 1.5 & 0 \\
\hline & 129.5 & $33 \%-67 \%$ & 2.0 & 0 \\
\hline \multirow{3}{*}{ 3- Pan in ice } & 64.5 & $17 \%-83 \%$ & 1.0 & 0 \\
\hline & 97 & $25 \%-75 \%$ & 1.5 & 0 \\
\hline & 129.5 & $33 \%-67 \%$ & 2.0 & 0 \\
\hline
\end{tabular}

In the second part of the experiments, flow visualization tests were conducted in a borosilicate square glass tray (2 mm wall thickness) with outside dimensions of $10 \times 10 \mathrm{~cm}$ and a depth of $5 \mathrm{~cm}$. A $9.6 \times 6 \times 3 \mathrm{~cm}$ ice wall placed on one side of the tray as shown with dark color in Fig. 1 (b). In order to create the ice wall without visual imperfections, directional freezing of demineralized water was utilized to prevent the inclusions of gas bubbles and other impurities. A 


\section{INTERNATIONAL OIL SPILL CONFERENCE}

bracket-shaped holder (shown with dashed line in Fig. 1(b)) was used to keep the ice wall fixed at the wall of the tray. A layer of methanol that was $3 \mathrm{~cm}$ thick with temperature of $10-12{ }^{\circ} \mathrm{C}$ (near flash point of methanol) was poured in the tray and then ignited with a propane torch.

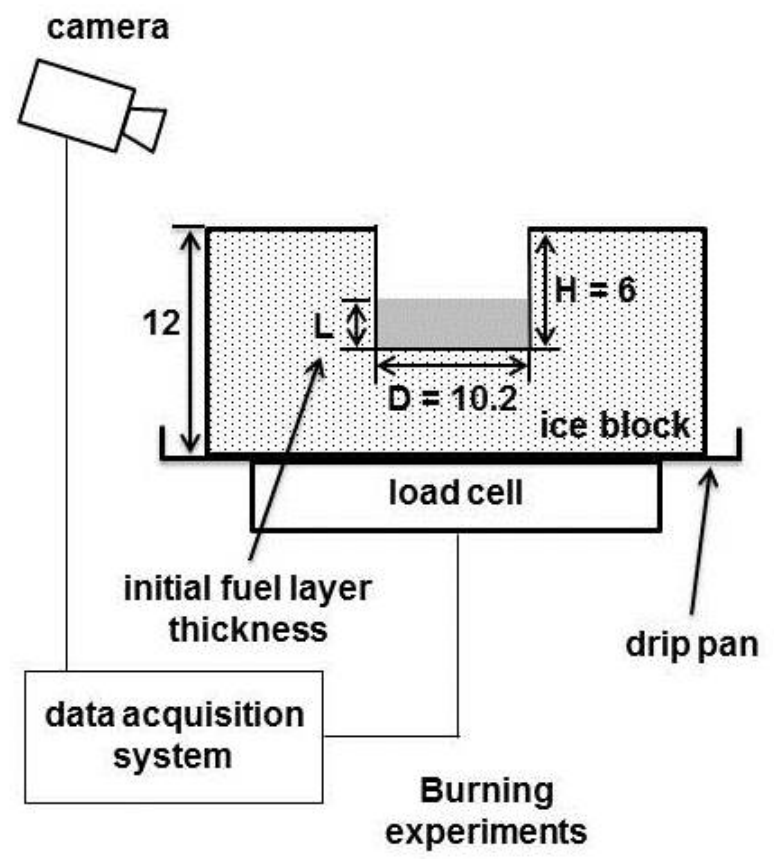

(a)

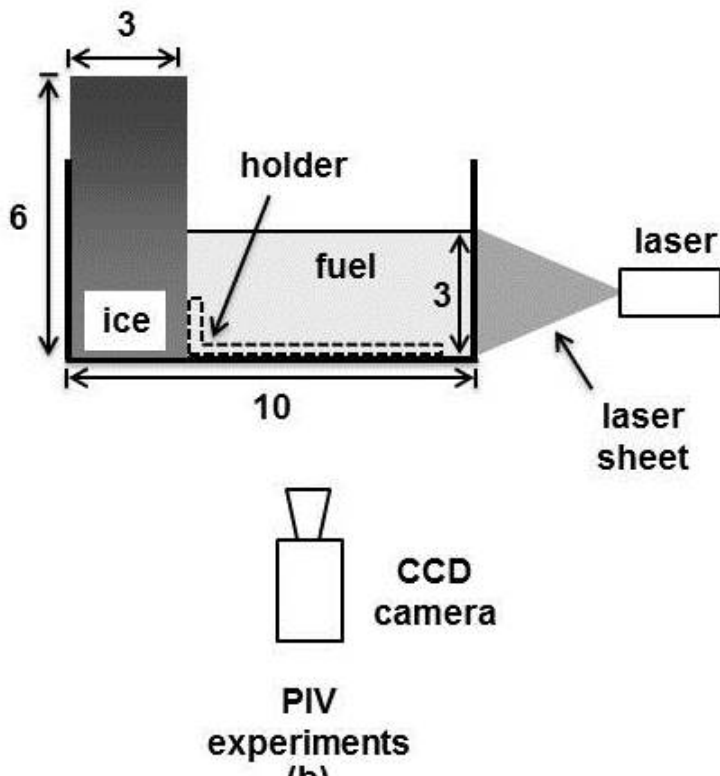

(b)

Figure 1. Schematic of the experimental setup. (a) Setup for burning behavior study (b) Setup for the flow visualization study with PIV system. The dimensions are in $\mathrm{cm}$ and not to scale.

The velocity field of the methanol layer on the midplane and perpendicular to the ice wall was obtained by PIV measurements (Laser: Nd:YAG NewWave Solo, 100 mJ/pulse, 532 nm, CCD Camera: HiSense 12 bit, $1280 \times 1024$ pixel resolution, Software: Flowmanager, Dantec Dynamics). The liquid fuel in the tray was seeded with $10 \mu \mathrm{m}$ silver coated hollow glass sphere particles. The particles specific gravity, relaxation time, and settling velocity were $1.1,0.12 \mu$ s and $0.25 \mu \mathrm{m} / \mathrm{s}$, respectively. The location of the laser sheet for the velocity measurements is shown in Fig. 1(b). The camera was placed perpendicular to the laser that illuminated a thin light sheet in the flow.

\section{RESULTS AND ANALYSIS}

Measurements of mass loss over time were analyzed to obtain the burning rate and burning 
Abstract ID: 2017-170

\section{INTERNATIONAL OIL SPILL CONFERENCE}

efficiency of methanol in the aforementioned conditions. The results are reported in Section 3.1. Results of the PIV study showing the flow field within the methanol is presented in Section 3.2. Finally, a discussion addressing the implications of in situ burning in icy condition is provided by the insight that is acquired from these experiments.

\subsection{Burning behavior and effect of ice}

The mass loss rates of methanol burning in the $10.2 \mathrm{~cm}$ diameter ice cavity are shown in Fig. 2. Experiments were repeated three times to ensure the repeatability of the measurements. There were two burning phases observed, first being the continuous increase to reach a peak and then a rapid decline to extinction. Similar trend was also reported previously for burning of ANS crude oil in a similar size ice cavity (Farahani, 2014) and also shown in Fig. 2 by the solid curve. The first phase where the burning rate is found to grow steadily was caused by expansion of pool fire due to melting of ice. The decline to extinction, however, was caused due to dilution of the methanol by the melt-water. In general, increase in the water content of methanol-water mixture reduces the mass loss rate of the mixture. This is because of the increase in the boiling point and latent heat of vaporization of the methanol-water mixture (Parag and Raghavan 2009).

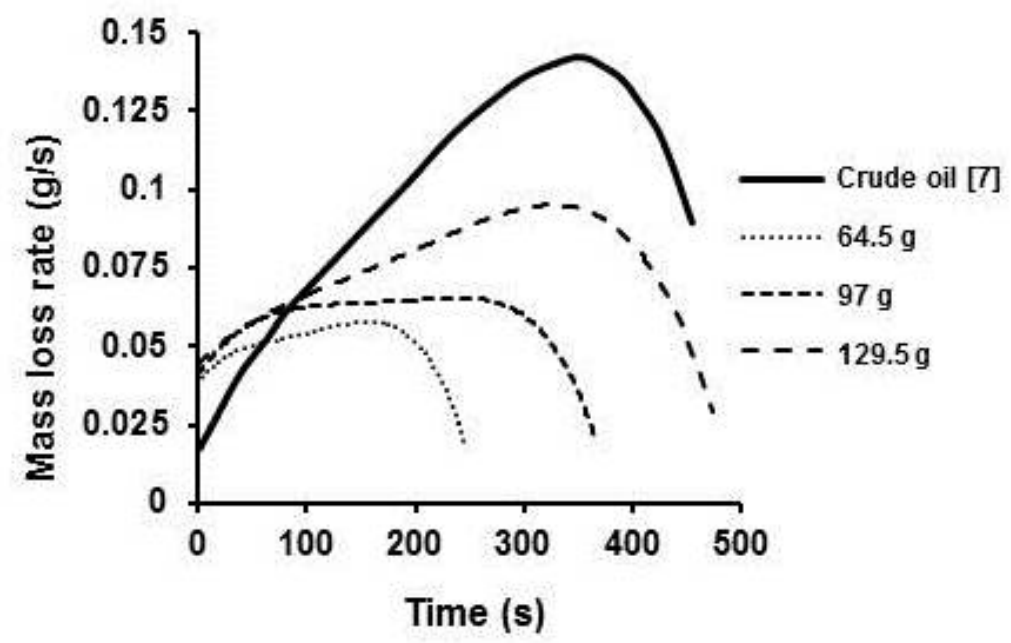

Figure 2. Mass loss rates for burning methanol in $10.2 \mathrm{~cm}$ diameter ice cavity. ANS crude oil mass loss rate in similar size cavity is added to the figure.

To observe the turnover of the ice shape for the tests in ice cavity, the ice block for each test 


\section{INTERNATIONAL OIL SPILL CONFERENCE}

was cut in half after extinguishment of the flame and an image was taken from the cross section. Figure 3 shows the change in the cavity geometry and labels the relevant dimensions associated with the geometry changes. The heat produced by the combustion of methanol melted the ice wall and created a semi-hemisphere void inside and around the circumference of the cavity (lateral cavity phenomena). The final diameter of the cavity measured at the location of the fuel layer was found to increase to $21 \mathrm{~cm}$ for a $2 \mathrm{~cm}$ layer of methanol equal to $129.5 \mathrm{~g}$. The increase in the diameter was more than two times the original diameter of the cavity. Also, due to accumulation of melt-water in the cavity, the liquid layer rose up. The penetration length of the fuel into the ice (lateral cavity) was found to be relatively small $(\sim 2 \mathrm{~cm})$ because of displacement of the liquid layer and short burning time ranging between 250 and 500 seconds. However, for a different scenario of burning, the penetration length could potentially increase significantly based on the type and amount of the fuel.

a) $64.5 \mathrm{~g}$

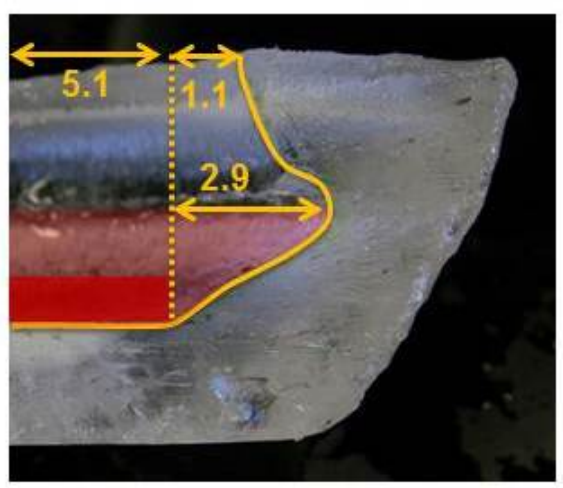

b) $96 \mathrm{~g}$

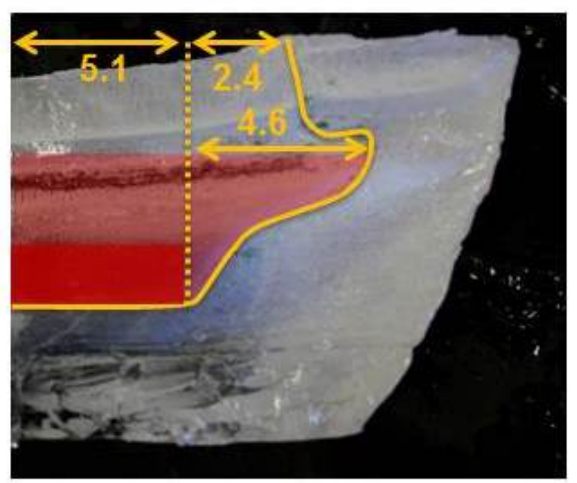

c) $129.5 \mathrm{~g}$

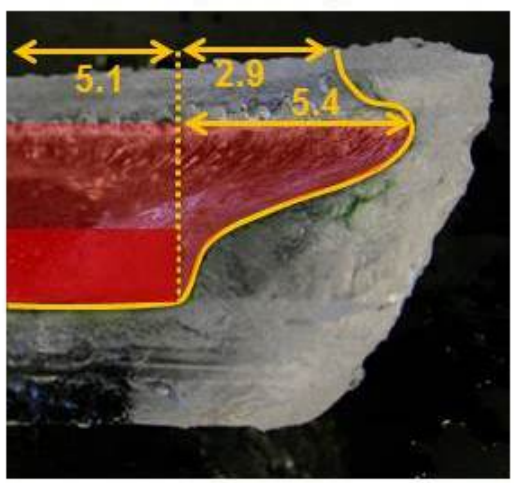

Figure 3.The change in form of the ice cavity after extinguishment of the flame. The vertical dashed line represents the location of the ice wall before ignition of methanol. The shaded area shows the initial and final methanol layer. Dimensions are reported in $\mathbf{c m}$.

To separate the effect of the cold boundary and ice melting, complementary experiments were conducted in a metal pan as explained in section 2. The mass loss rates of mixtures of methanol-water surrounded by water (at $20^{\circ} \mathrm{C}$ ) and ice bath (at $0{ }^{\circ} \mathrm{C}$ ) are shown in Figure 4 (a) and (b)), respectively. The mass loss rates for these two cases showed a short period of growth after ignition, following a long period of steady burning which ended in a rapid decline before extinction of the flame. Overall the tests in the pan burned for a longer duration as their dilution 


\section{INTERNATIONAL OIL SPILL CONFERENCE}

process was shorter compared to the ice cavity tests where melting of the ice was a significant source for dilution. The enclosure of the pan in the ice bath resulted in reduction of the mass loss rate for the three methanol-water ratios tested herein. In particular, the average mass loss rate of 17\%-83\% case (lowest methanol content) reduced from 0.047 to $0.026 \mathrm{~g} / \mathrm{s}$. The average mass loss rates of all the tests are reported in Fig. 4(c).

Comparing Fig.2 and Fig, 4 also shows that burning in ice cavity shows the highest amount of mass loss rate, although the total mass lost is relatively low. The increase in the mass loss rate is merely due to the widening process of the cavity which provides more surface area for the fuel to burn. When the average mass loss rates values per unit area of the fuel surface are analyzed, as shown in Fig. 4(d), it is clear that, the burning on ambient temperature water shows the highest average mass loss rate. For the tests in ice cavity, the mass loss rates were normalized based on the continuous increase in the diameter of the burning area. It was assumed that the expansion of the burning area followed a linear trend from initial to final diameter. As shown in Fig. 4(d), the normalized values of average mass loss rate in cold boundaries were significantly lower than the experiments in normal ambient.
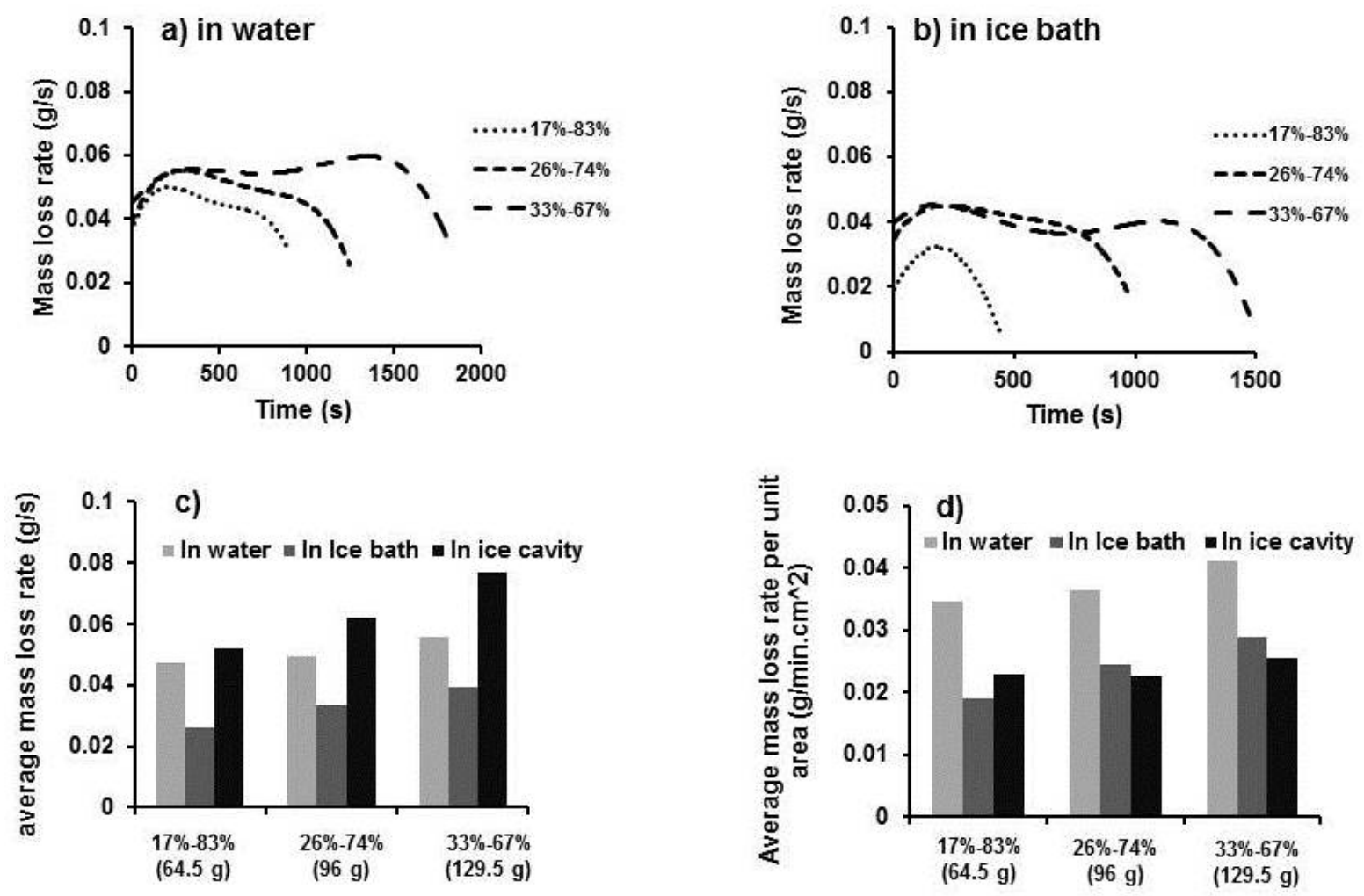
Abstract ID: 2017-170

\section{INTERNATIONAL OIL SPILL CONFERENCE}

Figure 4. Mass loss rate trends for burning on methanol-water mixture a) in water, b) in ice, c) average mass loss rate of all experiments d) Average mass loss rate per unit area of all experiments.

To better understand the burning behavior, burning efficiency (defined as \% of fuel consumed during burning), are calculated from the mass loss data and are presented here. Burning efficiencies of the tests are reported in Fig. 5. The highest burning efficiency (lowest amount of remaining fuel), was found to belong to the burning of methanol in water ( $70 \%)$, while the experiments in the ice bath showed lower burning efficiency percentages in general ( 40\%) due to influence of the ice bath surrounding the pan. Moreover, the burning efficiencies obtained from the tests in ice cavity show the lowest values ( 20\%). In addition to the cold boundaries, rapid dilution of methanol by melt-water seems to have a large impact on burning. Given the fact that initially there was no water in the ice cavity experiments, higher values of burning efficiency were expected. Yet, the burning efficiency values for the experiments in ice cavity were found to be the lowest.

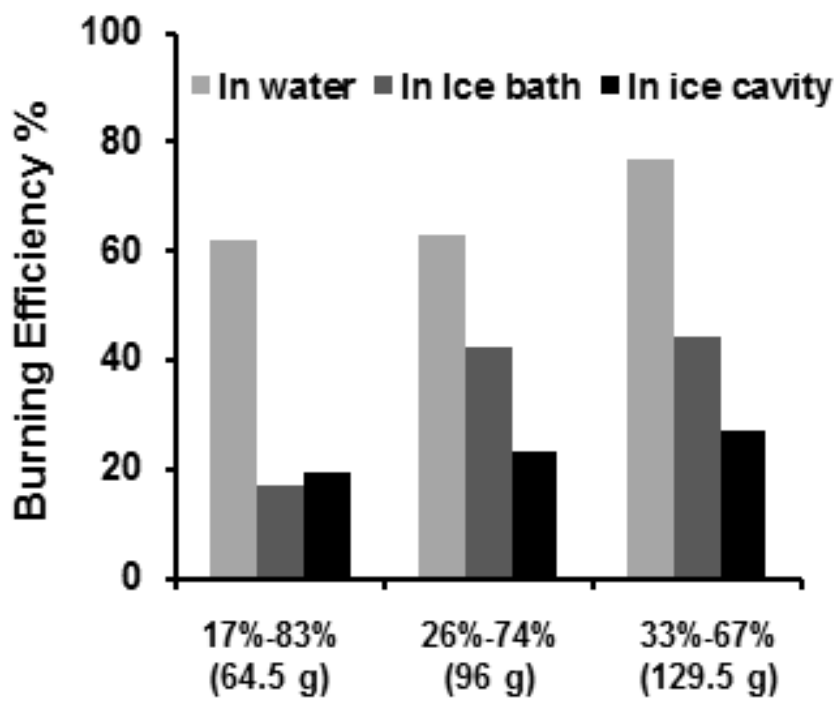

Figure 5. a) Burning efficiencies, b) Average mass loss rate per unit area, obtained from burning methanol-water mixtures in $10.2 \mathrm{~cm}$ diameter pan in 1- Room temperature water, 2Ice bath, and 3- Methanol in $10.2 \mathrm{~cm}$ diameter ice cavity.

Flammability (combustibility) of a given liquid fuel is based on the amount of vapor that is released from its surface to create a burning mixture with air (American Institute of Chemical 
Abstract ID: 2017-170

\section{INTERNATIONAL OIL SPILL CONFERENCE}

Engineers, 1996). When the fuel vapor concentration goes below a Lower Flammable Limit (LFL), the flame will extinct. For a miscible mixture of fuel-water, the degree of the flammability depends on its temperature and also the water content of the mixture (Liaw and Chiu 2006). Also, reduction of burning rate is reported for burning of alcohol-water mixtures as the water content increases (Parag and Raghavan 2009). In the current experiments, the water content increased as methanol burned away, hence diluting the mixture. In the tests in ice cavity, the dilution process was more significant due to melting of the ice, so the dilution point at which the mixture is rendered as non-combustible was reached faster. The presence of ice and addition of the melt-water also can change the flow field in the methanol layer. This will be discussed in the next section.

\subsection{Flow field near an ice wall}

For the second part of this study, the velocity field of methanol on the vertical midplane prependicular to the ice wall was measured by the PIV system described in section 2. Similar to the observations of the previous studies (Farahani et al. 2014, Farahani et al. 2015), the melting of the ice was more significant in the regions of the methanol layer contact with the ice wall versus regions of flame impingement. This led to the splitting and falling of the top section of the ice into the pool. The splitting of the ice, which happened consistently $125 \pm 5$ seconds after ignition, was used as an end-point to the PIV experiments. The presence of the ice and warm fuel in an icy pool fire creates a thermally mixed field that is assumed to be the driving force for convection in the liquid.

The buoyant and surface tension forces can be considered as the driving forces generated by a thermal gradient between the hot fuel layer and cold boundaries. In the areas near the ice wall a vertically downward flow is expected to occur because of buoyancy. Additionally, a horizontal temperature gradient will be present because of the presence of the ice producing a surface tension variation horizontally. Because surface tension is a decreasing function of temperature for methanol, a variation of the surface tension produces a flow (also known as Marangoni convection) on and below the free surface. The flow field 20 seconds after ignition of methanol showing a vortex near the ice wall is shown in Fig. 6. Figure 6 (a) and (b) show the velocity vector field 


\section{INTERNATIONAL OIL SPILL CONFERENCE}

overlaid on the velocity magnitude map and streamlines of flow with background map of vorticity magnitude, respectively. The maximum velocity magnitude of $6 \mathrm{~mm} / \mathrm{s}$ was found below the free surface and near the ice. The streamlines of flow showed a group of vectors below the surface traveling toward the ice which is induced by Marangoni convection. Also, the vortex appearing near the ice wall had a vorticity magnitude of less than $4 s^{-1}$. The pattern of the flow and the convective roll formed in the liquid fuel after ignition corroborates the hypothesis that was proposed in a previous study by the authors (Farahani et al. 2015).
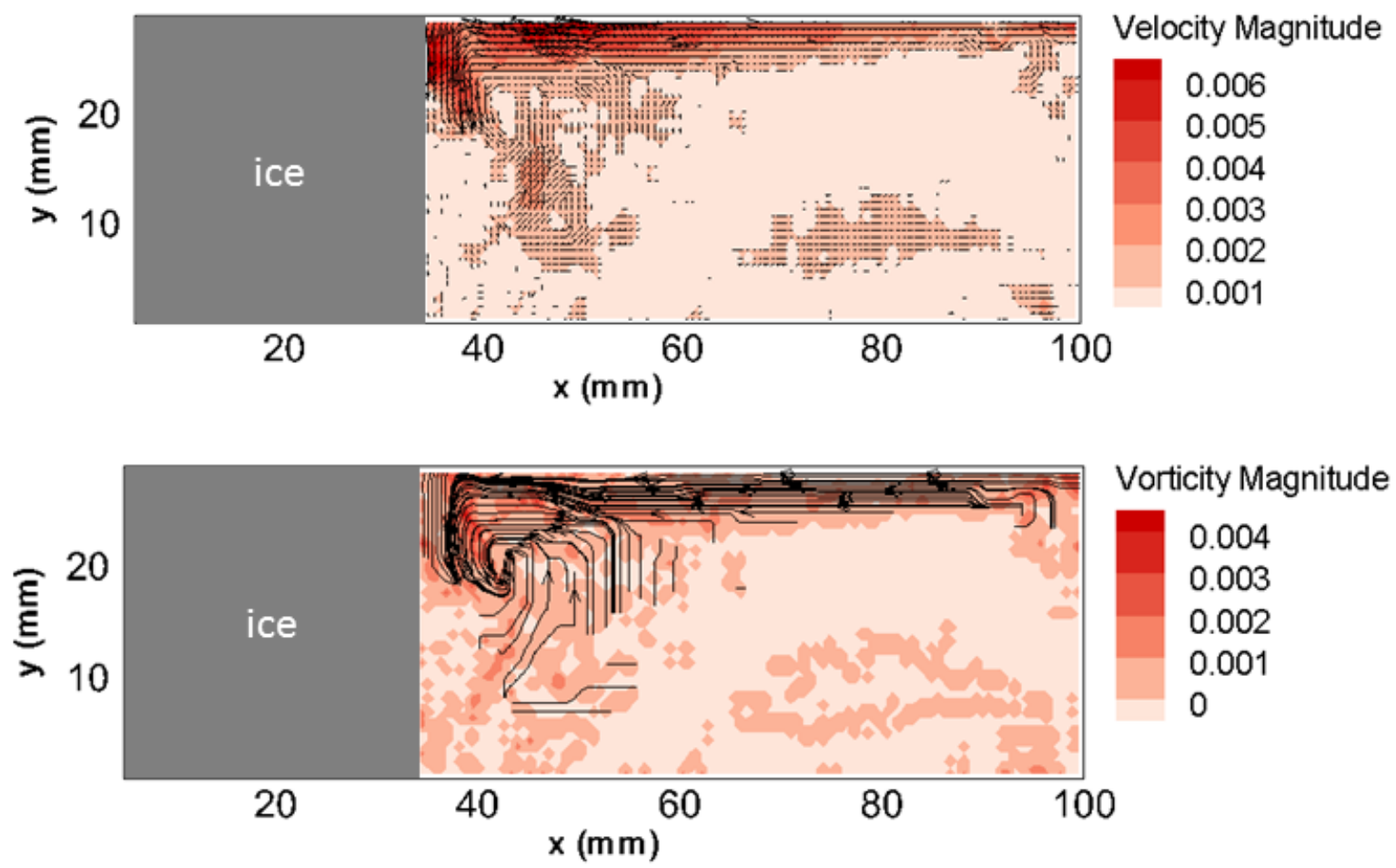

Figure 6. Flow field of methanol 20 seconds after ignition a) The vector field with background color map of velocity magnitude $(\mathrm{m} / \mathrm{s}) \mathrm{b}$ ) Streamlines of flow with background color map of vorticity magnitude $\left(s^{-1} \times 10^{-3}\right)$.

The flow field 100 seconds after ignition of methanol is presented in Fig. 7. Figure 7 (a) and (b) show the velocity vector field overlaid on the velocity magnitude map and streamlines of flow with background map of vorticity magnitude, respectively. At this stage the convective flows were not limited to the top layer of liquid and higher velocities were observed to occur in deeper areas. Velocities have increased to a maximum manitude of $1 \mathrm{~cm} / \mathrm{min}$ as shown in Fig. 7 (b). Convective flows in small-scale methanol pool fires are found to be affected by the thermal boundaries of the 


\section{INTERNATIONAL OIL SPILL CONFERENCE}

pool fires (Vali et al. 2014, Vali et al. 2016). However, the addition of melt-water is another factor that could alter the flow field of methanol. When the melting process begins, the melt-water flows downward alongside the ice wall due to its higher density. This generates a drag force for an immiscible fuel in water (e.g. alkanes). For a miscible fuel such as methanol, the melt-water also dilutes the mixture as it can mix with water. However, the tendency of melt-water is to sink because of its lower temperature and higher density compared to that of methanol. The sinking of the methanol-water mixture creates a downward flow which is counterbalanced by an upward flow toward the ice generating a counter-clock flow cycle as shown in Fig. 7. The vortex seen in the middle of the pool in Fig. 7 (b) is caused by the mentioned flow cycle.
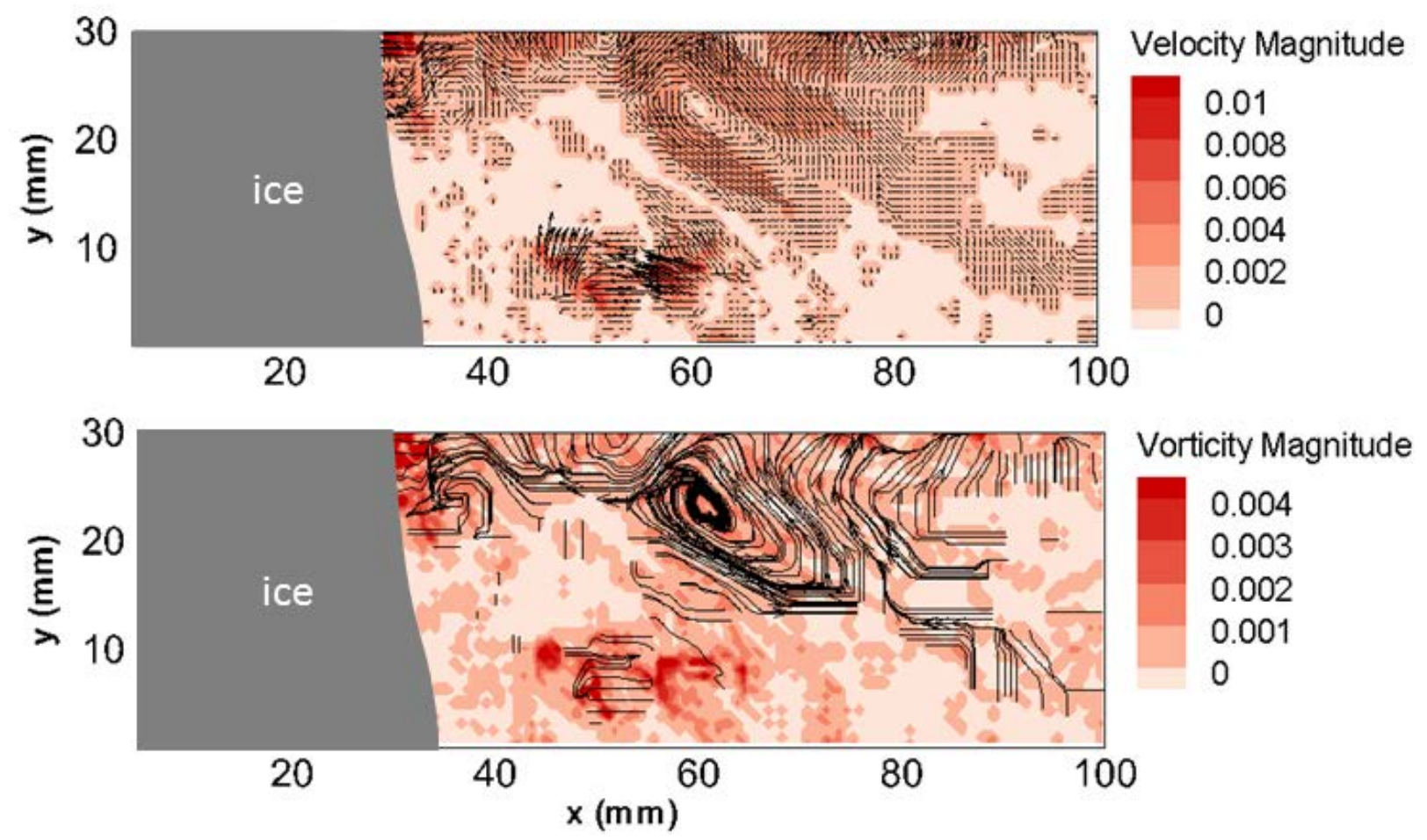

Figure 7. Flow field of methanol 100 seconds after ignition a) The vector field with background color map of velocity magnitude $(\mathrm{m} / \mathrm{s}) \mathrm{b}$ ) Streamlines of flow with background color map of vorticity magnitude $\left(s^{-1} \times 10^{-3}\right)$.

The PIV measurements showed two flow patterns in the 2 minutes burning duration of the experiments. In the first 30 seconds after ignition, a laminar surface flow from the center towards the ice wall was most evident and the rest of methanol layer was relatively still. The movement of the hot fuel towards the ice wall, promoted melting of ice leading to a larger counter-clockwise 
Abstract ID: 2017-170

\section{INTERNATIONAL OIL SPILL CONFERENCE}

vortex generation. This is the main contributing factor in diluting the methanol as more melt-water is introduced to the system and a likely factor in reducing the flammability of methanol-water mixture burning in an ice cavity burning in an ice cavity.

\subsection{Implications for spills in ice infested regions}

In situ burnings of spills on or adjacent to ice bodies are a more complicated practice compared to other type of in situ burns. Once ice is introduced to the problem, there are two main alterations in the in situ burning process. First, the geometrical parameters related to ice and oil changes due to melting of ice (parameters such as diameter of the pool, thickness of the oil layer, and ullage). Second, the presence of ice with very low temperatures, compared to the burning oil, brings about thermal instabilities that influence the burning of the fuel.

The changes in geometry of the problem occur due to the melting of the ice, both by accumulation of melt-water under the oil that displaces the fuel layer and also deformations of the ice such as widening of the pool by melting (e.g. lateral cavity). In more details, for a closed bottom pool (spills on solid ice), the oil layer elevates if the amount of melt-water is more than the amount of oil that is removed by burning and vice versa. In general, the smaller size pool brings more melting and less burning of the oil level that translates to faster accumulation of the melt-water and more vertical displacement of the fuel layer. A larger pool, however, tends to have higher regression and less melting thus the oil level will not vary significantly during the in situ burn. This condition increases the chance of having a deep lateral cavity. The consequences of such vertical movements in oil pools can vary from extinguishments because of overflow or causing a secondary unwanted fire. However, if the oil is in open bottom pools (e.g. spill in slush, brash, drift and pack ice), then the level of the oil will not be affected by the ice melting or regression of the oil. In this case a lateral cavity phenomenon is likely to reach its maximum length. The adverse effects of lateral cavity formation on the efficiency of the in situ burning are discussed in length (Farahani et al. 2014, Farahani, et al. 2015).

The thermal effects of low temperature boundaries on a lab-scale methanol fire were discussed in section 3.1. It was shown that burning efficiencies are significantly reduced when 
Abstract ID: 2017-170

\section{INTERNATIONAL OIL SPILL CONFERENCE}

burning in cold boundaries. However, larger pools of crude oil in ice ( $1 \mathrm{~m}$ diameter) have been observed to burn as efficient as a similar sizes pools in a metal pan (Shi et al. 2016). For spills in brash ice, the contact surface of the oil with ice increases with increasing in the ice content. This is a cause for significant heat losses, regardless to the size of the spill. Nevertheless, the key parameter affecting in situ burning with the presence of ice is the imposed temperature gradients on the oil layer by the presence of ice. The variation of temperature in the fuel layer has been found to be the driving mechanism for convection in the oil (Farahani et al. 2015). These convective flows are described to be the main cause for formations of lateral cavity. Previous studies have shown a lateral cavity of $\sim 10 \mathrm{~cm}$ length (Shi et al. 2016) in a 1m by $1 \mathrm{~m}$ square pit comprised of icy walls with $20 \%$ of fuel mass encapsulation. Potentially, many parameters could change the size of a lateral cavity and these parameters are currently under investigation. These parameters include viscosity, boiling point, and thermal diffusivity of the oil, ambient condition (cross wind) and burning time to name a few.

\section{CONCLUSIONS}

A series of experiments were conducted to add to the current understanding of the burning of liquid fuels in icy mediums. The burning behaviors of methanol pool fires in different conditions were studied to understand how the ice affects the burning. The mass loss rates and burning efficiency are primarily reduced when a cold boundary is imposed on the fuel layer. However, mass loss rates are found to be higher for burning in the ice cavity because of the melting of the ice and expansion of the cavity. Still, the lowest burning efficiency is found to belong to the burn tests in ice cavity even though these experiments started with no water content. The increase in water content is found to be the main reason for the early extinguishment of the flame and the decrease in burning efficiency. The velocity field of methanol was measured by the PIV system and the results of the measurements showed two different flow patterns in the 2 minutes burning duration of the experiments. In the first 30 seconds after ignition, the laminar flow near the surface induced by Marangoni convection was most evident and the rest of methanol layer was relatively still. After this initial period, the enhanced melting and sinking of the cold melt-water initiated a flow cycle in the tray. This is the main contributing factor in diluting the methanol as more 
Abstract ID: 2017-170

\section{INTERNATIONAL OIL SPILL CONFERENCE}

melt-water is introduced to the system and a likely factor in reducing the flammability of methanol-water mixture.

\section{Acknowledgement}

We thank Jianhua Fan and Simon Furbo for letting use their PIV system, and Ulises Alva for his helps with the experiments. This study was partially funded by the Bureau of Safety and Environmental Enforcement (BSEE), US Department of the Interior, Washington, DC, under Contract Number E14PC00010. Hamed Farmahini Farahani and Grunde Jomaas were supported by the Danish Council for Independent research (Grant DDF - 1335-00282). The contents do not necessarily reflect the views and policies of the BSEE, nor does mention of the trade names or commercial products constitute endorsement or recommendation for use.

\section{REFERENCES}

I. Buist and D. Dickins (1987). Experimental spills of crude oil in pack ice. International Oil Spill Conference, American Petroleum Institute.

I.A. Buist, et al. (2013). In Situ Burning in Ice-Affected Waters: State of Knowledge Report, JIP. 7.1.1.

P. J. Brandvik, et al. (2006). Short state-of-the-art Report on Oil Spills in Ice-Infested Waters. Oil in Ice, SINTEF Materials and Chemistry.

P. W. Bellino, M.R. Flynn, and A. S. Rangwala. (2013). "A study of in situ burning of crude oil in an ice channel." Proc. Combust. Inst. 34(2): 2539-2546.

E. DeCola, T. Robertson, S. Flethcer, S Harvey (2006). Offshore Oil Spill Response in Dynamic Ice Conditions, Nuka Research and Planning Group, LLC; Harvey Consulting, LLC.

H. Farmahini Farahani, G. Jomaas, A. S. Rangwala. (2015). "Effects of convective motion in n-octane pool fires in an ice cavity." Combustion and Flame 162(12): 4643-4648.

H. Farmahini Farahani, X. Shi, A. Simeoni, A. S. Rangwala. (2014). "A study on burning of crude oil in ice cavities." Proc. Combust. Inst. 35(3): 2699-2706.

A. Hamins , S. Fischer, T. Kashiwagi , (1999). A global model for predicting the burning rates of liquid pool fires.

A. Hamins , S. Fischer, T. Kashiwagi, (1994). "Heat Feedback to the Fuel Surface in Pool Fires." Combustion Science and Technology 97(1-3): 37-62.

H. J. Liaw, and Y. Y. Chiu (2006). "A general model for predicting the flash point of miscible mixtures." Journal of hazardous materials 137(1): 38-46. 
Abstract ID: 2017-170

\section{INTERNATIONAL OIL SPILL CONFERENCE}

A. Nakakuki, (1994). "Heat Transfer in Small Scale Pool Fires." Combustion and Flame 96: 311-324.

A. Nakakuki, (1994). "Heat Transfer Mechanisms in Liquid Pool Fires." Fire Safety Journal 13: 339-363.

S. Parag, and V. Raghavan (2009). "Experimental investigation of burning rates of pure ethanol and ethanol blended fuels." COMBUSTION AND FLAME 156(5): 997-1005.

S. L. Ross (2003). Tests to Determine the Limits to In Situ Burning of Thin Oil Slicks in Broken Ice, Report to Minerals Management Service and ExxonMobil Upstream Research. Herndon, VA, U.S.

X. Shi, P. W. Bellino, A. Simeoni, A. S. Rangwala (2016). "Experimental study of burning behavior of large-scale crude oil fires in ice cavities." Fire Safety Journal 79: 91-99.

A.Vali, et al. D. S. Nobes, and L. W. Kostiuk (2013). "Effects of altering the liquid phase boundary conditions of methanol pool fires." Experimental Thermal and Fluid Science 44: 786-791.

A.Vali, D. S. Nobes, and L. W. Kostiuk (2014). "Transport phenomena within the liquid phase of a laboratory-scale circular methanol pool fire." COMBUSTION AND FLAME 161(4): 1076-1084.

A.Vali, D. S. Nobes, and L. W. Kostiuk (2016). "Characterization of flow field within the liquid phase of a small pool fire using particle image velocimetry technique." Experimental Thermal and Fluid Science 75: 228-234.

L. Van Gelderen, N. L. Brogaard, M. X Sorensen,J. Fritt-Rasmussen, A. S. Rangwala,G. Jomaas(2015). "Importance of the slick thickness for effective in-situ burning of crude oil." Fire Safety Journal 78: 1-9.

C. L. Yaws, (1995). Handbook of Thermal Conductivity, Volume 3: Organic Compounds C8 to C28, Gulf Professional Publishing.

T. Yumoto, A. Takahashi, T. Handa (1997). "Combustion Behavior of Liquid Fuel in a Small Vessel: Effect of Convective Motion in the Liquid on Burning Rate of Hexane in the Early Stage of Combustion." COMBUSTION AND FLAME 30: 33-43.

American Institute of Chemical Engineers. Center for Chemical Process Safety. Guidelines for use of vapor cloud dispersion models. Wiley-AIChE, 1996. 